\title{
EDITORIAL
}

\section{Mechanism of ICU-acquired weakness: muscle contractility in critical illness}

\author{
Jane Batt ${ }^{*}$, Sunita Mathur ${ }^{2}$ and Hans D. Katzberg ${ }^{3}$
}

๑) 2017 Springer-Verlag Berlin Heidelberg and ESICM

Weakness is common after an episode of critical illness and its associated disability may persist for years, with significant negative impact on the survivor [1]. Strength depends on both the presence and normal function of skeletal muscle, so that either muscle wasting or impaired contractility (muscle-specific force) can induce weakness. This is best appreciated in the gerontology literature where sarcopenia, the concept of which has evolved from simple muscle wasting to loss of muscle mass and function, and dynapenia (loss of strength and power not necessarily associated with loss of muscle mass) are identified and considered separately because they are not synonymous [2]. In aging, the decline in strength exceeds the loss of muscle mass and this is attributed to changes throughout the neuroaxis including those occurring at the peripheral nerve in addition to muscle. Muscle unloading/inactivity in animal models promotes a loss of contractile proteins that exceeds the loss in muscle cell size, resulting in decreased myocyte-specific force [3] and in young adults muscle atrophied by immobilization may recover to regain mass but not strength, reinforcing the notion that, like the dynapenia of aging, muscle strength and mass may be discordant. A pilot study of critical illness-associated weakness also suggests this holds true [4]. Therefore, studies evaluating ICU-acquired weakness (ICUAW) should ensure complete assessment for peripheral neuropathy, neuromuscular junction dysfunction in addition to evaluation for myopathy-loss of muscle mass and deficiency of intrinsic contractility. Although the clinical phenotype may be weakness, the relative contributions of underlying processes and cellular and molecular mechanisms may be very different. This review

\footnotetext{
*Correspondence: Jane.batt@utoronto.ca

${ }^{1}$ Department of Medicine, Keenan Centre for Biomedical Research, St. Michael's Hospital, University of Toronto, Toronto, ON, Canada

Full author information is available at the end of the article
}

will focus on the impairment of muscle contractility in critical illness and discuss the responsible muscle-derived cellular and molecular mechanisms.

If present, critical illness polyneuropathy or neuromuscular blockade will decrease the force-generating capacity of muscle in the ICU patient. Similarly, myonecrosis and/or altered muscle composition will decrease musclespecific force. Even in the absence of these phenomena cellular signalling within the intact muscle fibers of critically ill patients can impede contractile function directly, with or without concurrent induction of changes in muscle size/mass, at least in the short term. Primary muscle contributions to impaired contractility are multifactorial, but appear to predominantly relate to three fundamental processes: (1) altered muscle membrane excitability, (2) excitation-contraction uncoupling, and (3) altered muscle bioenergetics (Fig. 1). It must be emphasized, however, that most research in these areas has been conducted in animal models of critical illness myopathy (CIM) and sepsis, and is supported by limited evidence from humans.

Abnormalities of muscle membrane excitability including decreased conduction velocity, increased relative refractory period, and reduced fiber excitability occur in the critically ill [5]. Animal models suggest the primary etiology is an acquired abnormality of the muscle sodium channel, induced in part by the inflammatory milieu of critical illness and treatment interventions, which influences both the baseline resting membrane potential and the depolarization in response to an action potential [6]. Interestingly, despite the importance of voltage-gated calcium channels and potassium channels to membrane excitability, these have been less studied. Abnormalities of the ryanodine and L-type $\mathrm{Ca}^{2+}$ channel function influencing muscle membrane excitability in critical illness models have been reported $[7,8]$.

\section{实



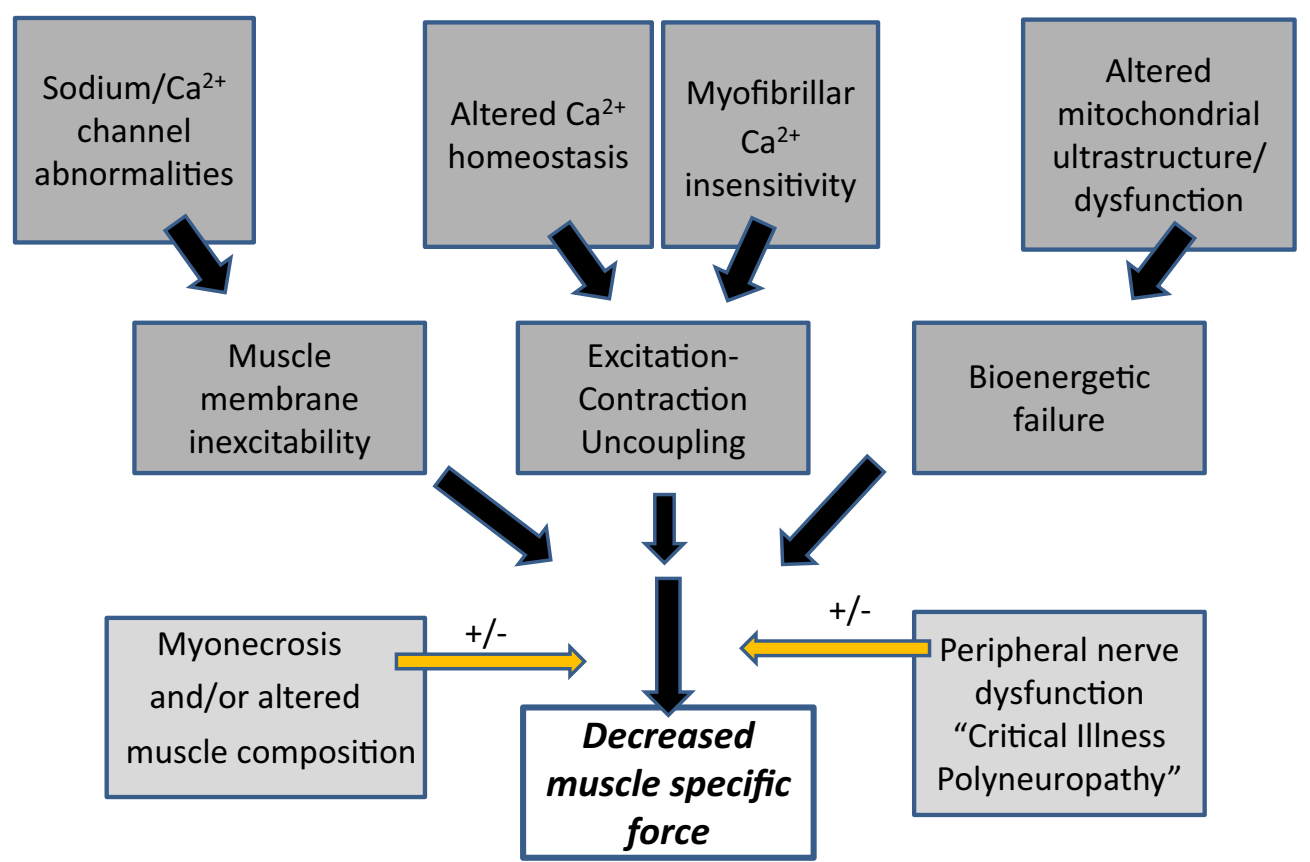

Fig. 1 Impaired muscle contractility in critical illness

Both skeletal and cardiac muscle excitation-contraction uncoupling, induced by altered intracellular calcium homeostasis, has been demonstrated extensively in sepsis [9]. Conversion of the motor neuron electrical signal to skeletal muscle mechanical contraction occurs by stimulation of $\mathrm{Ca}^{2+}$ release from the sarcoplasmic reticulum (SR) into the cytosol, where it enables contraction. Abnormalities in calcium handling, due to altered membrane receptors/ion channels (i.e., ryanodine receptor, SERCA1) and altered myofilament $\mathrm{Ca}^{2+}$ sensitivity, contribute to the excitation-contraction uncoupling in rodent models of sepsis and CIM $[10,11]$.

Bioenergetic compromise in critical illness contributes to muscle fatiguability and weakness. Mitochondrial ultrastructural damage and dysfunction are evident in skeletal muscle in animal models of critical illness (sepsis, burns) and critically ill patients, resulting in muscle ATP depletion and cytopathic hypoxia $[9,12]$. In a recent long-term rat model of ICUAW that demonstrates a decline in muscle specific force and atrophy, the perturbation of genes that regulate mitochondrial dynamics appears to contribute to the myopathy [13]. Conversely, restoration of mitochondrial function in muscle satellite cells of septic mice increases muscle specific force [14]. Mitochondrial biogenesis evaluated in the muscle of critically ill patients has been found to be both up- and downregulated. Upregulated muscle mitochondrial biogenesis may be associated with survival, but studies have not evaluated the correlation between mitochondrial biogenesis and strength in humans [15].

While all of these aforementioned processes will impair muscle contractility, regardless of the status of muscle mass, the cellular signalling networks that influence contraction and myofiber size are often intertwined. For example, the pro-inflammatory milieu of critical illness will both impair muscle contractility and induce muscle wasting. TNF- $\alpha$ activates reactive oxygen species which directly interfere with myofibril function [16] by uncoupling excitation and contraction. TNF- $\alpha$ also promotes muscle proteolysis by the ubiquitin proteasome system (UPS) [17], thereby inducing muscle atrophy. Interleukin-1 (IL-1) is a cytokine biomarker that correlates with outcomes in critical illness. In animal models, IL-1 induces muscle atrophy [18] and alters calcium transients in the SR thereby inducing excitation-contraction uncoupling [19]. Genetic manipulation of mitochondrial fission machinery alters mitochondrial morphology, with subsequent dysfunction, energy stress, and downstream activation of muscle atrophy networks [20].

Given the complexity and intricacy of the linkages in the cellular signalling networks influencing muscle contractility and size, one might predict a markedly heterogeneous phenotype of weakness in the critically ill patient and survivor. When one also considers the potential for differential muscle repair and regrowth following injury and degradation during critical illness, it becomes apparent that long-term muscle dysfunction is likely not 
a single entity, but a constellation of variable degrees of muscle wasting and compromised specific force. To date, the research agenda for CIM has focused largely on muscle wasting. This needs to change as it is clear from research conducted across varied disease states and healthy aging that muscle excitability, contractility, and mass must each be evaluated as their contributions to the weakness phenotype may be discordant; all must be reconstituted for complete recovery to be realized.

\section{Author details}

${ }^{1}$ Department of Medicine, Keenan Centre for Biomedical Research, St. Michael's Hospital, University of Toronto, Toronto, ON, Canada. ${ }^{2}$ Department of Physical Therapy, University of Toronto, Toronto, ON, Canada. ${ }^{3}$ Department of Medicine, University Health Network, University of Toronto, Toronto, ON, Canada.

\section{Compliance with ethical standards}

\section{Conflicts of interest}

On behalf of all authors, the corresponding author states that there is no conflict of interest.

Received: 3 January 2017 Accepted: 16 February 2017

Published online: 3 March 2017

\section{References}

1. Herridge MS, Chu LM, Matte A et al (2016) The RECOVER Program: disability risk groups and one year outcome after 7 days of mechanical ventilation. Am J Respir Crit Care Med 194:831-844. doi:10.1164/ rccm.201512-23430C

2. Manini TM, Clark BC (2012) Dynapenia and aging: an update. J Gerontol A Biol Sci Med Sci 67:28-40. doi:10.1093/gerona/glr010

3. Larsson L, Li X, Berg HE, Frontera WR (1996) Effects of removal of weightbearing function on contractility and myosin isoform composition in single human skeletal muscle cells. Pflugers Arch Eur J Physiol 432:320-328

4. Dos SC, Hussain SN, Mathur S et al (2016) Mechanisms of chronic muscle wasting and dysfunction after an intensive care unit stay. A pilot study. Am J Respir Crit Care Med 194:821-830. doi:10.1164/ rccm.201512-23440C

5. Trojaborg W (2006) Electrophysiologic techniques in critical illness-associated weakness. J Neurol Sci 242:83-85. doi:10.1016/j.jns.2005.11.019

6. Rich MM, Pinter MJ (2003) Crucial role of sodium channel fast inactivation in muscle fibre inexcitability in a rat model of critical illness myopathy. J Physiol 547:555-566. doi:10.1113/jphysiol.2002.035188
7. Kraner SD, Wang Q, Novak KR, Cheng D, Cool DR, Peng J, Rich MM (2011) Upregulation of the CaV 1.1-ryanodine receptor complex in a rat model of critical illness myopathy. Am J Physiol Regul Integr Comp Physiol 300:R1384-R1391. doi:10.1152/ajpregu.00032.2011

8. Friedrich $\mathrm{O}$, Hund $\mathrm{E}$, von Wegner $\mathrm{F}$ (2010) Enhanced muscle shortening and impaired $\mathrm{Ca}^{2+}$ channel function in an acute septic myopathy model. J Neurol 257:546-555. doi:10.1007/s00415-009-5362-5

9. Friedrich $\mathrm{O}$, Reid MB, Van den Berghe G, Vanhorebeek I, Hermans G, Rich MM, Larsson L (2015) The sick and the weak: neuropathies/ myopathies in the critically ill. Physiol Rev 95:1025-1109. doi:10.1152/ physrev.00028.2014

10. Llano-Diez M, Cheng AJ, Jonsson W, Ivarsson N, Westerblad H, Sun V, Cacciani N, Larsson L, Bruton J (2016) Impaired Ca ${ }^{2+}$ release contributes to muscle weakness in a rat model of critical illness myopathy. Crit Care 20:254. doi:10.1186/1471-2164-12-602

11. Callahan LA, Nethery D, Stofan D, Dimarco A, Supinski G (2001) Free radical-induced contractile protein dysfunction in endotoxin-induced sepsis. Am J Respir Cell Mol Biol 24:210-217. doi:10.1165/ajrcmb.24.2.4075

12. Latronico N, Bolton CF (2011) Critical illness polyneuropathy and myopathy: a major cause of muscle weakness and paralysis. Lancet Neurol 10:931-941. doi:10.1016/S1474-4422(11)70178-8

13. Corpeno KR, Salah H, Gastaldello S et al (2016) Mechano-signalling pathways in an experimental intensive critical illness myopathy model. J Physiol 594:4371-4388. doi:10.1113/JP271973

14. Rocheteau P, Chatre L, Briand D et al (2015) Sepsis induces long-term metabolic and mitochondrial muscle stem cell dysfunction amenable by mesenchymal stem cell therapy. Nat Commun 6:10145. doi:10.1038/ ncomms 10145

15. Carre JE, Orban JC, Re L et al (2010) Survival in critical illness is associated with early activation of mitochondrial biogenesis. Am J Respir Crit Care Med 182:745-751. doi:10.1164/rccm.201003-03260C

16. Hardin BJ, Campbell KS, Smith JD, Arbogast S, Smith J, Moylan JS, Reid MB (2008) TNF-alpha acts via TNFR1 and muscle-derived oxidants to depress myofibrillar force in murine skeletal muscle. J Appl Physiol 104:694-699. doi:10.1152/japplphysiol.00898.2007

17. Li YP, Lecker SH, Chen Y, Waddell ID, Goldberg AL, Reid MB (2003) TNF-alpha increases ubiquitin-conjugating activity in skeletal muscle by up-regulating UbcH2/E220k. FASEB J 17:1048-1057. doi:10.1096/ f. $02-0759$ com

18. Cooney R, Owens E, Jurasinski C, Gray K, Vannice J, Vary T (1994) Interleukin-1 receptor antagonist prevents sepsis-induced inhibition of protein synthesis. Am J Physiol 267:E636-E641

19. Friedrich $\mathrm{O}$, Yi B, Edwards JN et al (2014) IL-1alpha reversibly inhibits skeletal muscle ryanodine receptor. A novel mechanism for critical illness myopathy? Am J Respir Cell Mol Biol 50:1096-1106. doi:10.1165/ rcmb.2013-00590C

20. Romanello V, Guadagnin E, Gomes L et al (2010) Mitochondrial fission and remodelling contributes to muscle atrophy. EMBO J 29:1774-1785. doi:10.3389/fphys.2015.00422 